\title{
Emerging infections in pregnancy
}

Keywords: paradox of pregnancy, fetal antigens, maternal-fetal interface, IOM, CDC, ebola viral disease

\section{Introduction}

One of the most intriguing puzzles in modern immunology involves the "paradox of pregnancy," in which immunologic tolerance to paternally derived fetal antigens is achieved despite an apparently adequate maternal defense against infection. With $50 \%$ of its genetic material derived from its father, the fetus's susceptibility to rejection by the maternal immune system is similar to the susceptibility of a transplanted organ. Evidence indicates that the maternal immune system may tolerate fetal antigens by suppressing cell-mediated immunity while retaining normal humoral immunity. ${ }^{1}$ These changes are known to occur locally at the maternal-fetal interface but may also affect systemic immune responses to infection. Although pregnant women are not immune suppressed in the classic sense, immunologic changes of pregnancy may induce a state of increased susceptibility to certain intracellular pathogens, including viruses, intracellular bacteria, and parasites.

As strategies to deal with emerging infectious disease threats are developed, a key component is consideration of special populations, including pregnant women. Several issues are relevant to infectious disease threats during pregnancy. First, changes in immunity and physiology during pregnancy may make pregnant women more susceptible to or more severely affected by infectious diseases. Second, the effects of infectious diseases on the fetus may be unknown and difficult to predict, and diagnosis of infection in the fetus or infant can be challenging. Third, prophylaxis and treatment appropriate for the general population might not be appropriate for pregnant women. ${ }^{2}$

The terms "emerging and reemerging diseases" were formally given by Joshua Lederberg, Robert B. Shope, and Mary Wilson in 1987. ${ }^{1}$ The term is used in reference to diseases of infectious origin and whose incidence in humans has either increased within the past two decades or threatens to increase in the near future. A 1992 report by the Institute of Medicine (IOM) on emerging infections, which underscored the microbial threats to health in the United States, provided the impetus for current widespread attention on emerging and reemerging infectious diseases. This formal designation seemed to have drawn greater attention and placed the issue of emerging diseases high on the agenda of national and international health programs and has formed a key part of various organizational, institutional, and departmental research focuses. ${ }^{1}$ The field of emerging disease exploration was strengthened by the creation of "special pathogens branch," which is a special force on emerging diseases by the WHO at the Centre for Disease Control and Prevention (CDC) in Atlanta, Georgia, USA. The main objective was to spearhead research and related activities aimed at understanding the emergence of new infectious diseases and their reappearance in new populations after a long period of silence and find ways to prevent or control them.

Specifically, factors affecting the environment include depletion of forests, expansion and modernization of agricultural practices, and natural disasters such as floods. These potentially lead to changes in microbial ecological niches and fuel microbial adaptation to human host. Sociodemographic factors such as increase in population density,
Volume 5 Issue 7 - 2017

\author{
Subhashish Das \\ Professor, Department of Pathology, Sri Devaraj Urs Medical \\ College, India
}

Correspondence: Subhashish Das, Professor, Department of Pathology, Sri Devaraj Urs Medical College, Kolar Tamaka, India, Tel 94808498।8, Email daspathology@gmail.com

Received: October 23, 2017| Published: December 29, 2017

falling living standards, decline of infrastructure, human travel, conflicts and social instability, and killing of wild animals for meat all lead to increase in host-microbe contact, which facilitate infections in humans. There are also some pathogens whose emergence is as a result of deliberate human action. These are those employed as biological weapons for destruction and so their emergence is "deliberate".

Besides host and environmental factors, changes or mutation in the genome of a pathogen, which occurs as a result of exposure to chemicals and antimicrobial agents (e.g., antibiotic), may lead to gene damage and emergence of drug resistant pathogen variants that could cause new disease. Thus, human, microbial, and environmental factors constitute major causes of infectious disease emergence and the virulence or pathogenic potential depends on a complex combination of these factors. However, generally, emerging infectious diseases caused by viral pathogens are responsible for the greatest proportion of the EID threat, having caused about two-thirds of the infectious disease burden and usually characterized by very high epidemics. Examples are Filoviruses, Ebola, and Marburg.

Emerging and neglected infectious diseases are a real public health threat, and infectious disease outbreaks can have serious social, political, and economic effects. Much has been learned from previous outbreak events and far-reaching advances have been made since the landmark IOM report, which underscored the important concept of emerging infectious diseases. Pandemic preparedness however remains a major global challenge. A complex number of factors relating to human behaviour and activities, pathogen evolution, poverty, and changes in the environment as well as dynamic human interactions with animals have been found to contribute to infectious disease emergence and transmission. Aggressive research is warranted to unravel important characteristics of pathogens necessary for diagnostics, therapeutics, and vaccine development and possibly enable detection of those pathogens with the potential to cause epidemic. National and international organizations networking, effective interagency and international research collaborations, appropriate financial support of the public health infrastructure, and poverty reduction are very vital for addressing emerging and neglected infectious disease threats.

In view of all these we are going to discuss 3 important emerging infections. Namely

I. Ebola viral diseases

II. Scrub typhus and

III. Zika Virus 
With particular reference to pregnancy as it involves the prognosis both mother and child and the society at large.

\section{Ebola viral disease (EVD) and pregnancy}

Ebola virus disease (EVD) was first recognized in 1976, with multiple subsequent outbreaks confined to sub-Saharan Africa. In 2014 a pandemic EVD noticed across Europe and US. The burden of EVD in pregnant women remains uncertain due to the low numbers of patients affected in previous outbreaks and limitations in data collection during the current epidemic. EVD is a filo virus infection, though to be transmitted to human from an unknown animal reservoir. Current knowledge suggests that bats or non-human primates represent the most likely species involved in the occurrence of sporadic human outbreaks.

There are five identified species of EVD: a) Zaire ebola virus, b) Sudan ebola virus, c) Tai Forest ebola virus, d) Bundibugyo ebola virus and e) Reston ebola virus. Zaire ebola virus is the most lethal strain and is responsible for the current epidemic in Western Africa.

Zoonotic transmission is believed to transfer Ebola from the animal reservoir to the human host through hunting and meat consumption practices, while human-to human transmission efficiently propagates EVD through mucosal contact with infected body fluids. ${ }^{2}$ The risk of transmission continues following death; hence, corpses remain at high risk and must be handled in accordance with full infection control procedure.

During 1976 EVD was occurred in Zaire with particular involvement of pregnant women. This increased transmission was traced back to poor infection control practices at an antenatal clinic where vitamin injections were being administered with the same syringe instead of pregnancy per se The management strategy for a woman who is suspected of having Ebola would normally to be isolate her, test her and treat medically until laboratory confirmation of her EVD status is received.

For women with obstetric emergencies requiring prompt operative action, this is problematic as invasive procedures frequently place health-care workers at increased risk of exposure to body fluids and may therefore be deemed too dangerous to perform to the exclusion of EVD. ${ }^{4}$ The similarities between the symptoms of a woman presenting with pregnancy-related complications and EVD is more complicated by the poor condition of antenatal healthcare in the affected states. Usually a woman comes to a health-care centre with late and multiple complications due to delays in recognizing the disease along with poor socio-economic status and limited infrastructural facilities.

The most distressing factor of ebola pathogenesis of EVD is the fact that the Ebola virus is able to cross the placenta and infect the amniotic fluid and fetus. Furthermore, after a woman has survived Ebola, and viraemia has resolved, the products of conception (POC) remain NAAT positive, frequently with results signifying high virus levels $^{5}$ (Figure 1)

\section{Scrub typhus}

Scrub typhus is an acute febrile illness caused by orientia tsutsugamushi transmitted to humans by the bite of the larva of trombiculid mites. Even though scrub typhus in pregnancy is uncommon, it is associated with increased foetal loss, preterm delivery, and small for gestational age infants. ${ }^{6}$ After an incubation period of 6-21days, onset is characterized by fever, headache, myalgia, cough and gastrointestinal symptoms.

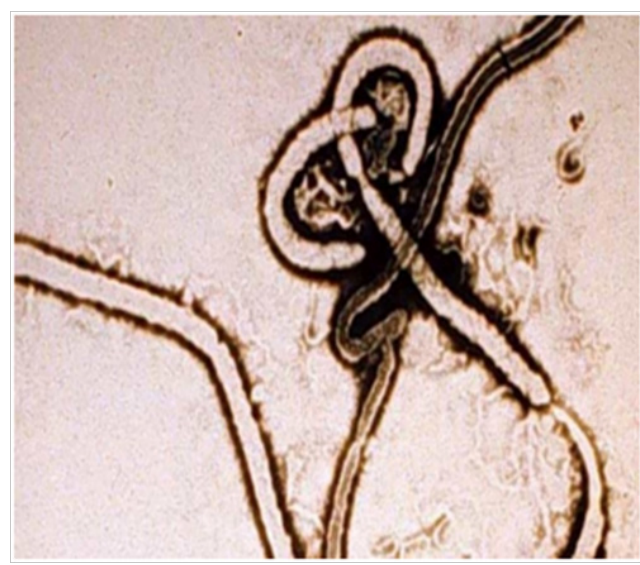

Figure I Ebola virus under electron microscope view.

The clinical manifestations of scrub typhus in pregnant women are similar to those of non-pregnant adults. Studies reported that scrub typhus in pregnancy may be associated with increased foetal loss, preterm delivery, and small for gestational age infants. There have been reports of vertical transmission from transplacental infection and transmission in perinatal blood-borne infection during labor causing neonatal scrub typhus in mother with acute febrile illness during pregnancy. Diagnosis of scrub typhus during pregnancy is same as in non-pregnant women and is based on an exposure history, clinical symptoms, and confirmed serological studies. The standard therapy for scrub typhus in non-pregnant adults is doxycycline and chloramphenicol. However, doxycycline, a class D drug (according to the U.S. Food and Drug Administration) has been associated with fetal risk and is contraindicated in pregnant women. One study reported a pregnant woman who had received chloramphenicol, and despite the mother's high scrub typhus-specific IgG and IgM titers, IgM was not detected in the neonate's sera. ${ }^{7}$ However, chloramphenicol, is classified as a class $\mathrm{C}$ drug and is prescribed with caution to late trimester pregnant women because of increased risk to the foetus at the time of delivery ${ }^{8}$ (Figure 2).

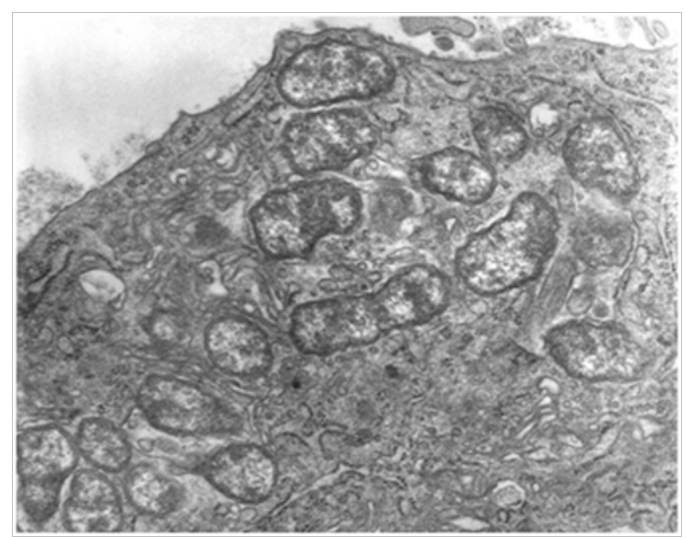

Figure 2 Scrub typhus under electron microscope.

\section{Zika virus}

Zika virus is an emerging mosquito-borne (Aedes genus) arbovirus of the Flaviviridae family. Unfortunately, despite an unprecedented surge in attempts to rapidly advance perinatal clinical testing for a previously obscure arbovirus, there are several ongoing hindrances to molecular-and sonographic-based screening and diagnosis of congenital Zika virus infection. These include the following: 
1. Difficulty in estimating the timing of exposure for women living in endemic areas and thus limited interpretability of immunoglobulin M serologies.

2. Cross-reaction of immunoglobulin serologies with other endemic flaviruses, such as dengue.

3. Persistent viremia and viruria in pregnancy weeks to months after primary exposure. and

4. Fetal brain malformations and anomalies preceding the sonographic detection of microcephaly.

Until recently Zika viral illness was thought to be self-limiting, resembling a mild version of dengue virus or chikungunya virus. Zika virus is transmitted via aedes spp of mosquitos and is spread via sexual transmission and vertical (mother to child) and blood transfusions. Clinical manifestations of Zika infection include fever, headache, arthralgia myalgia, and maculopapular rash, although only 1 of every 4-5 people who are infected manifest symptoms. Thus, clinical symptoms are not an effective screening tool for the diagnosis or relative risk of Zika infection because approximately $80 \%$ are likely asymptomatic. The exact Zika virus pathogenesis is known because of multiple reasons first, we do not know the risk estimates for fetal infection or CNS abnormalities in the setting of either maternal or amniotic fluid infection as measured by rRT-PCR. Second, there are multiple strains of Zika virus and the majority of Zika virus strains isolated in the Americans are of Asian lineage, and all are associated with fetal congenital infection. Third, the exact mechanism of Zika virus entry into fetal compartment has not been defined. ${ }^{9}$ Multiple lines of evidence suggest that placental cells, including placental macrophages, are permissive to replication. Fourth maternal viremia and viruria has been observed to persist much beyond even after the termination of pregnancy.

The diagnostic accuracy of tests employed in the ZIKV context is still unclear because existing studies are based on epidemiological models. In some women, ZIKV positivity was confirmed later in pregnancy; although the standard RT-PCR tests done in early trimesters were negative. Fetal ultrasound tests that were negative in the first and second trimesters were positive in late pregnancy stage. ${ }^{10}$ The absolute risk of developing fetal abnormalities is also unclear, but microcephaly is unlikely being a rare condition.

The broad spectrum of clinical presentation and complications in fetuses and newborns of Zika virus-infected pregnant women provides an insight into the potential liability for affected women, their families and health systems in resource-constrained settings. The possibility of an asymptomatic presentation proposes an emphasis on routine antenatal care and attention to signs of fetal brain abnormalities by health providers in pregnant women with molecular or epidemiological links to Zika virus infection. ${ }^{11}$

Ongoing research on live animal models may help to understand the pathogenesis of Zika virus, with a potential for application to exposed pregnant cohorts. The rapid rate of emerging data from research on Zika virus infections in pregnancy and recent years proposes a need for frequent updates. As newer studies become available, further research on adverse pregnancy outcomes and implications on longterm consequences of Zika virus during pregnancy will further enable early diagnosis and better management modalities in provider care. Furthermore, personal protective measures should be encouraged (Figure 3). ${ }^{12}$

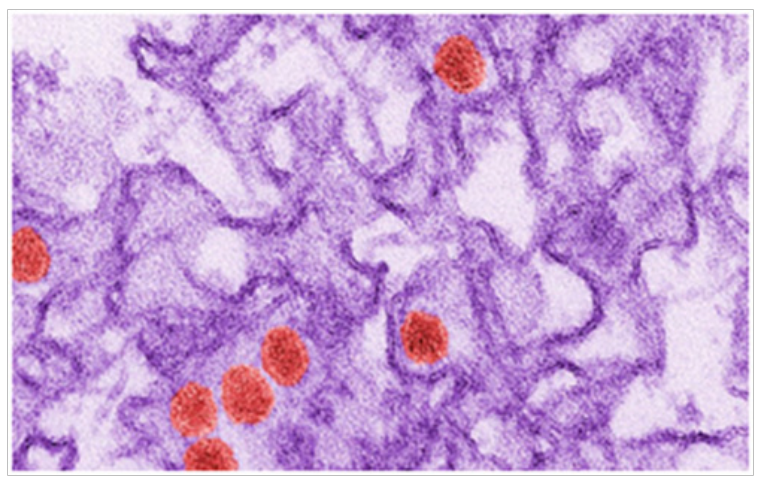

Figure 3 Zika virus under electron microscope view.

\section{Conclusion}

Future directions in research to decrease the risk of infectious processes associated with blood transfusion include the development of blood substitutes, increased utilization of autologous blood, generation of cellular blood components from omnipotent stem cell lines, and improvement in blood testing and transfusion practices based on epidemiological studies. Because many transfusion decision-making processes are made in the perioperative period, surgeons and anesthesiologists must keep abreast of current practices and perspectives in this rapidly progressing field. ${ }^{1}$

As public health professional respond to emerging infections, particular attention needs to be paid to pregnant women might be more susceptible to, or more severely affected by, emerging infections. The effects of a new maternal infection on the embryo or fetus are difficult to predict. Some medications recommended for prophylaxis or treatment could harm the embryo or fetus. ${ }^{2}$

Compared with what is known about conventional disease threats, knowledge about currently recognized emerging infectious diseases is quite limited. Soon we will likely be faced with novel pathogens about which little or nothing is known. Because the effects of emerging infections in pregnant women might differ from those in the general population, pregnancy must be considered a potential risk factor for disease susceptibility as well as for illness and death. Unfortunately, pregnancy issues are often not well addressed in outbreak investigations, ongoing prospective studies, or emergency preparedness planning. ${ }^{1} \quad$ Future scientific inquiry and medical investigations must include pregnancy-related issues as a vital component.

A carefully planned public health approach, which includes input from individuals with expertise in pediatrics, obstetrics and infectious diseases, will improve our ability to protect women and their offspring from adverse consequences associated with emerging. ${ }^{11}$

\section{Acknowledgements}

None.

\section{Conflict of interest}

The author declares no conflict of interest.

\section{References}

1. Beeching NJ, Fenech M, Houlihan CF. Ebola virus disease. BMJ. 2014;349:7348. 
2. Fletcher T, Fowler RA, Beeching NJ. Understanding organ dysfunction in Ebola virus disease. Intensive Care Med. 2014;40(12):1936-1939.

3. Jamieson DJ, Uyeki TM, Callahan WM, et al. What every obstetrician should know about Ebola. Obstet Gynecol. 2014;24(5):1005-1010.

4. Black BO. Obstetrics in the time of Ebola: challenges and dilemmas in providing lifesaving care during a deadly epidemic. BJOG. 2015;122(3):284-286.

5. Baggi FM, Taybi A, Kurth A, et al. Management of pregnant women infected with Ebola virus in a treatment centre in Guinea. Surveill. 2014;19(49):20983.

6. Mandell GL, Bennett JE, Dolin R. Principles and Practice of Infectious Diseases. 6th ed. USA: Churchill Livingstone; 2005. p. 2309-2310.

7. Mathai E, Rolain JM, Verghese L, et al. Case reports: scrub typhus during pregnancy in India. Trans R Soc Trop Med Hyg. 2003;97(5):570-572.
8. Wang CL, Yang KD, Cheng SN, et al. Neonatal scrub typhus: a case report. Pediatrics. 1992;89(5 Pt 1):965-968.

9. Duffy MR, Chen TH, Hancock WT, et al. Zika virus outbreak on Yap Island, federated states of Micronesia. NEngl J Med. 2009;360(24):25362543.

10. Gatherer D, Kohl A. Zika virus: A previously slow pandemic spreads rapidly through the Americas. J Gen Virol. 2016;97(2):269-273.

11. Paixao ES, Barreto F, da Gloria Teixeira M, et al. History, Epidemiology, and Clinical Manifestations of Zika: A Systematic Review. Am J Public Health. 2016;106(4):606-612.

12. Cao-Lormeau VM, Musso D. Emerging arboviruses in the Pacific. Lancet. 2014;384(9954):1571-1572. 